\title{
PRÁTICAS EDUCATIVAS PARENTAIS: AUTONOMIA E EXPRESSÃO DE AFETO
}

\author{
Maria de Fátima Minetto \\ fa.minetto@gmail.com \\ Ana Caroline Bonato da Cruz \\ Universidade Federal do Paraná
}

Fecha de Recepción: 12 Marzo 2018

Fecha de Admisión: 10 Abril 2018

\section{RESUMO}

0 desenvolvimento da criança está relacionado a qualidade das relações familiares. Com o nascimento de uma criança com deficiência, a família pode experenciar sentimentos negativos interferindo na relação pai-filho, repercutindo nas práticas educativas parentais. Esta pesquisa pretendeu descrever práticas educativas com ênfase em autonomia e expressão de afeto utilizadas por pais com filhos com Atraso Significativo no Desenvolvimento. 0 método incluiu análises descritivas e inferências. Participaram 86 famílias, sendo 45 formadas por crianças com desenvolvimento típico e $41 \mathrm{com}$ crianças com atraso significativo no desenvolvimento. Todos os participantes preencheram o Inventário de Práticas Educativas Parentais (CRPR). Os resultados permitiram concluir que há diferença estatisticamente significativa entre os pais de crianças com desenvolvimento típico e os pais de crianças com Atraso Significativo no Desenvolvimento na escolha de práticas educativas, em relação ao uso do controle autoritário $(F(119)=3,61, p<0,05)$, autonomia $(F(119)=5,37$, $p<0,01)$, e na expressão de afeto $(F(119)=6,10, p<0,01)$. Concluiu-se que pais com filhos com Atraso Significativo no Desenvolvimento precisam de rede de apoio eficiente e orientações sobre práticas educativas mais adequadas para o desenvolvimento dos filhos. Sugere-se a criação de grupos de apoio que possam fornecer orientações de práticas educativas mais adequadas para este público.

Palavras-chave: práticas educativas parentais, deficiência intelectual, autismo, autonomia, afeto

\section{ABSTRACT}

Parental educational practices: autonomy and expression of affection.

Children's development is related to the quality of the family relationships. When a children with disabilities births the family can experiment negatives feelings as affliction and frustration. The way how parentes deal with this crisis influences on the educational practices that they will perform with their children. The purpose of this study is to descrive education practices of parentes with empha- 


\section{PRÁTICAS EDUCATIVAS PARENTAIS: AUTONOMIA E EXPRESSÃO DE AFETO}

sis on autonomy and affection expression utilized by parentes of childrens with developmental delay. The method includes descriptive and inferential analysis. Participated 86 families wich 45 has children with typical development and 41 has children with developmental delay (childrens without phenotypes but with intelectual disabilities light or moderate, or autism diagnosis). The results shows that there's a significant statistical difference between the parents with children with typical development and the children's parents with developmental delay about the choice of educational practices, in what concerns on the authoritarian control $(F(119)=3,61, p<0,05)$, stimulation of autonomy $(F(119)=5,37, p<0,01)$, and affection expression $(F(119)=6,10, p<0,01)$. The datas allow to conclute that parentes with childrens with developmental delay need a eficient support network and orientation about educational practices more appropriates to the childrens development. Unproportional use of authoritarian control associated with difficult on affection expression are educational practices that do not contribute to the development of a children. Creating support groups for parents with children with developmental delay could be a way to provide orientation about educational practices more apropriates.

Keywords: educational practices of parents; intelectual disability; autism; autonomy; affection

\section{INTRODUÇÃO}

A família é um sistema que proporciona aos indivíduos noção de pertencimento assim como fornece apoio para o desenvolvimento humano. Este sistema se desenvolve em movimentos contínuos de equilíbrio-adaptação e desequilíbrio, como resposta a mudanças internas e externas que alteram seu funcionamento. (Minuchin, Fishman, 1990). Uma das mudanças externas que interfere no desenvolvimento de algumas famílias é a identificação de uma criança com deficiência (Buscaglia, 2006). 0 que nem sempre é fácil, pois, em alguns casos, a variabilidade da manifestação do transtorno é muito grande e/ou não há presença de características físicas aparentes (Minetto, 2010). Associado a tal fator, ainda há o fato de 0 desenvolvimento infantil ser multifatorial, integral e continuo; características que dificultam a mensuração do atraso no desenvolvimento infantil (Organização Pan-Americana, 2005).

Quando a família recebe um diagnóstico de atraso do desenvolvimento de uma criança deparase com o que Franco (2015) referiu como "crise": uma mudança que não há possibilidade de volta a situação anterior, por tanto acompanhará a família para sempre. Cada família lida com tal momento de uma forma particular, sendo importante que esta consiga se reestruturar e demonstrar flexibilidade diante mudanças (Buscaglia, 2006; Minuchin, 1982). Em especial porque estas crianças precisam, tanto quanto qualquer outra, de pais e mães que estejam disponíveis emocionalmente e que possam oportunizar interação, cuidado, amor e envolvimento a seus filhos (Franco, 2016).

Diversos autores pontuam a importância da família na promoção do desenvolvimento da criança (Bronfenbrenner, 1996; Szymanski, 2004; Fiamengui e Messa, 2007). Segundo Darling e Steinberg (1993) os comportamentos que os pais direcionam aos filhos com as funções de educação e socialização são compreendidos como práticas educativas. Estudos realizados sobre o tema têm identificado que a forma como as práticas educativas parentais são apresentadas na família tem efeitos sobre cada membro da família (Stasiak, Weber e Tucunduva, 2014; Weber et al, 2006) assim como eventos intra e extrafamiliares afetam as escolhas de práticas educativas parentais (Szymaski, 2004). Segundo Weber (2017) práticas educativas positivas (entendidas como a participação do pais da vida dos filhos) estão relacionadas a melhor autoestima dos filhos; assim como a ausência de regras e monitoria são características de risco para depressão e comportamentos disruptivos.

Um dos estudos teóricos de relevância nesta área são os de Baumrind (1966), que diz que a maneira pela qual os pais educam seus filhos é determinante para que os mesmos tenham comportamentos considerados socialmente adequados. Para a autora pode-se classificar os estilos 
parentais em três modelos: 1) Pais Autoritativos (são aqueles que tentam direcionar as atividades de seus filhos de maneira racional e orientada, incentivando o diálogo e compartilhando com a criança o raciocínio e as consequências que existem na forma como ela se comporta. Exercem firme controle nos pontos de divergência, colocando a sua perspectiva de adulto, sem restringir a criança. Pais Autoritativos preparam seu filho para a autonomia); 2) Pais Autoritários (Priorizam a obediência como uma virtude e são a favor de medidas punitivas para lidar com os comportamentos da criança que consideram inadequados, exigem sempre o máximo e somente pensam em como seu filho vai ser no futuro); 3) Pais Indulgentes (tentam se comportar de maneira não punitiva e passiva frente aos comportamentos dos seus filhos, evitando confrontações, buscam a realização de seus desejos, existe superproteção em relação a dificuldades).

Já outros autores como Maccoby e Martin (1983) fizeram uma releitura do modelo de Baumrind e dividiram 0 estilo indulgente em dois tipos: 0 indulgente (permissivo, atende todos os desejos da criança) e o negligente (não reconhecem ou atendem as necessidades da criança). Para os autores supra citados dois aspectos são determinantes a exigência e a responsividade. A exigência consiste no grau de monitoramento que os pais têm sobre o comportamento do filho. A responsividade inclui a capacidade dos pais em perceber e considerar os pontos de vista da criança. Assim pais responsivos são amorosos e envolvidos com as necessidades da criança. Weber (2009) diferencia 0 envolvimento integral dos pais do comportamento intrusivo, entendendo que o segundo consiste em não aceitar nem respeitar as escolhas do filho. Tal diferenciação faz-se importante, visto que, ainda de acordo com a autora a educação de qualidade engloba proporcionar momentos nos quais a criança experimente fazer as coisas por si mesma e suas próprias escolhas. De forma que também é uma educação para a autonomia.

Diante do panorama descrito, a apresente pesquisa tem como objetivo descrever práticas educativas com ênfase em autonomia e expressão de afeto utilizadas por pais com filhos com Atraso Significativo no Desenvolvimento; tendo como objetivos específicos a) identificar os aspectos que envolvem a autoridade nas práticas educativas parentais; b) Identificar os aspectos que envolvem expressão de afeto nas práticas educativas parentais.

\section{MÉTODO}

A presente pesquisa caracteriza-se como transversal, pois analisa um momento em particular da história dos participantes. Seu caráter é exploratório, descritivo e explicativo; uma vez que se apoiou em entrevistas com pessoas que vivenciaram (ou vivenciam) a temática investigada; ocupou-se em descrever as características dos fenômenos observados, e também em correlacionar tais fenômenos (Gil, 1999; Sampieri, Collado e Lucio, 1994).

\section{Participantes}

Participaram da pesquisa 86 famílias das quais 45 são pais/mães de crianças com desenvolvimento típico (Grupo 1); e 41 são pais/mães de crianças com atraso significativo no desenvolvimento (Grupo 2). As crianças que compuseram o Grupo 2 não apresentavam características fenotípicas evidentes, mas possuíam laudos que comprovam deficiência intelectual leve ou moderada, conforme critérios diagnósticos estabelecidos pelo CID-10 (Classificação Internacional de Doenças Décima Revisão), a saber:

- CID-10: F70.1 - Retardo mental leve - comprometimento significativo do comportamento, requerendo vigilância ou tratamento.

- CID-10: F71.1 - Retardo mental moderado - comprometimento significativo do comportamento, requerendo vigilância ou tratamento. 


\section{PRÁTICAS EDUCATIVAS PARENTAIS: AUTONOMIA E EXPRESSÃO DE AFETO}

\section{Instrumentos}

Foram utilizados dois instrumentos para a realização da coleta de dados: Questionário Sociodemográfico e Inventário de Práticas Educativas Parentais (CRPR).

0 Questionário Sociodemográfico foi elaborado por uma das autoras da pesquisa, a fim de obter informações características dos participantes como: faixa etária, gênero, escolaridade, situação conjugal, idade da criança, existência ou não de diagnóstico, e outros.

0 Inventário de Práticas Educativas Parentais (CRPR - Child Rearing Practices Report) foi desenvolvido por Block (1965 apud Dekovic, Janssens e Gerris, 1991) e passou por adaptações realizadas por outros autores (Dekovic, 1989; Dekovic, Janssens e Gerris, 1991). 0 objetivo deste inventário é descrever atitudes, valores, objetivos de pais e mães na educação de seus filhos. Ele é composto por seis categorias de análise divididas de acordo com o perfil de práticas educativas. 0 Perfil Autoritário contempla: o controle autoritário (comandos diretos, imposições, proibições, etc), supervisão da criança (o pai/mãe acompanhar o que a criança faz); indução e controle da ansiedade (ameaças verbais ou não verbais sobre a consequência de seus atos). 0 Perfil Autoritativo engloba as seguintes características: orientação consistente (incentivo positivo, orientações, sugestões), estimulação e encorajamento à autonomia (incentivar tomada de decisões e 0 conhecimento das consequências de cada ato); liberdade e expressão de afeto (possibilitar que tanto os pais como as crianças falem e expressem seus sentimentos).

A versão utilizada nesta pesquisa refere-se ao instrumento traduzido e adaptado semanticamente por Minetto (2010), uma vez que inventário não é adaptado para a população brasileira, e a autora encontrou bons índices de Cronbach, sugerindo boa qualidade do instrumento. Esta adaptação brasileira foi embasada na versão holandesa do instrumento (Dekovic, Janssens e Gerris, 1991), sendo um questionário em formato de escala de Likert composto por 35 itens.

\section{Procedimentos}

A presente pesquisa foi submetida e aprovada pelo Comitê de Ética em Pesquisa com Seres Humanos da Universidade Federal de Santa Catarina (CEPSH/UFSC) (Certificado no 193).

Após a aprovação do projeto de pesquisa pelo Comitê de Ética, foi feito contato com Escolas de Educação Especial e Escolas de Ensino Regular da região sul do país. Neste momento a pesquisa foi apresentada, bem como os procedimentos necessários para a sua realização. Os pais das escolas participantes foram contatados e convidados a fazer parte do estudo. Todos os pais que aceitaram assinaram o Termo de Consentimento Livre e Esclarecido e agendaram dia a horário para a aplicação dos instrumentos. Os participantes puderem optar por levar o material para casa e entregar na escola ou responder na escola em sala preparada para esta atividade. 0 tempo de preenchimento dos instrumentos variou entre 20 e 30 minutos.

As instituições que aceitaram participar receberem, após o término da pesquisa, palestras com os resultados obtidos, a fim de auxiliar na promoção do desenvolvimento infantil e na elaboração de estratégias de intervenção.

\section{Análise de dados}

Os dados da presente pesquisa foram analisados por meio de estatística descritiva (frequências, médias, desvio padrão) e também análises inferenciais (testes paramétricos). As informações obtidas através dos instrumentos foram codificadas e tratadas no programa estatístico SPSS-15.0 for Windows (Statistical Package For The Social Sciences). 


\section{RESULTADOS E DISCUSSÃO}

Inicialmente serão apresentadas as informações de caracterização dos grupos de participantes, obtidas através das informações fornecidas nos questionários sociodemográficos. A análise descritiva dos dados mostrou que em relação aos participantes que compõem o Grupo 1: 23 crianças eram do gênero feminino e 22 do gênero masculino; a média de idade das crianças foi 6,42 anos $(D P=2,28)$ Em relação aos pais das crianças: a média da idade da mãe foi de 34,87 anos $(D P=8,19)$ e a média de idade do pai foi de 36,31 (DP=8,28); e a escolaridade predominante tanto entre as mães como entre os pais foi ensino médio completo (51,1\% e $46,7 \%$, respectivamente). A maioria $(66,7 \%)$ das famílias encontrava-se em situação conjugal casada e com renda familiar de dois a cinco salários mínimos (55,6\%).

A composição do Grupo 2 foi de 30 crianças do gênero masculino e 11 do gênero feminino, com idade média de 6,95 anos ( $D P=2,11)$, sendo que a maioria $(81,55)$ das crianças frequentava escola especial. Em relação aos pais, a média da idade das mães foi de 35,71 anos (DP=7,96) e a média de idade dos pais foi de 37,27 anos $(\mathrm{DP}=7,45)$. A maioria dos pais e das mães declarou como escolaridade 0 ensino médio completo ( $39 \%$ e $43,9 \%$ respectivamente). Nestas famílias a composição familiar predominante foi casada $(80,5 \%)$, com a renda familiar predominante de dois a cinco salários mínimos (46,3\%).

Para a análise das respostas indicadas através da escala Likert do Inventário de Práticas Educativas Parentais (CRPR) foi realizada inicialmente estatística descritiva (média e desviopadrão). Os dados estão apresentados na Tabela 1. Pode-se observar que tanto o Grupo 1 apresentou maior consistência em suas características de práticas educativas, com predomínio de práticas autoritativas, uso de reflexão, incentivo à autonomia e externalização do afeto. Já os participantes do Grupo 2, apesar de também ser caracterizado por média maior no que se refere as práticas autoritativas; faz pouco uso de reflexões, assim como não expressam muito afeto. A fim de verificar a existência de diferenças estatísticas significantes os dados foram comparados entre grupos par a par a partir do Teste de Análise de Variância (Anova).

Tabela 1 - Média e Desvio Padrão das dimensões do Instrumento Práticas Parentais nos dois grupos de análise.

\begin{tabular}{|c|c|c|}
\hline $\begin{array}{c}\text { Dimensões das Práticas } \\
\text { Parentais }\end{array}$ & $\begin{array}{c}\text { Grupo } 1 \\
\text { (Desenvolvimento } \\
\text { Típico) }\end{array}$ & $\begin{array}{c}\text { Grupo 2 } \\
\text { (Atraso significativo } \\
\text { do } \\
\text { Desenvolvimento) }\end{array}$ \\
\hline Autoridade & $163,02$ (D.P. $=13,85)$ & $153,98$ (D.P. $=16,67)$ \\
\hline Supervisão & $58,91$ (D.P. $=6,73)$ & $57,93$ (D.P. $=8,26)$ \\
\hline Indicador de ansiedade & $15,13$ (D.P. $=1,82)$ & $14,15$ (D.P. $=2,65)$ \\
\hline Total autoritário & $23,29$ (D.P. $=3,34)$ & $21,46($ D.P. $=5,17)$ \\
\hline Reflexão & $97,33$ (D.P. $=10,30)$ & $93,54$ (D.P. $=12,58)$ \\
\hline Autonomia & $25,51($ D.P. $=3,00)$ & $22,83($ D.P. $=4,89)$ \\
\hline Ex. afeto & $45,27$ (D.P. $=4,45)$ & $41,39$ (D.P. $=6,45)$ \\
\hline Total autoritativo & $36,51$ (D.P. $=4,26)$ & $34,63($ D.P. $=3,65)$ \\
\hline
\end{tabular}

A partir da análise estatística observou-se diferença estatisticamente significativa na dimensão Autoridade entre os dois grupos $(F(119)=3,61 ; p<0,05)$. Tal dado indica que os pais e mães do Grupo 1 fazem maior uso de controlo autoritário (como comando diretos, repreensões, etc) que os pais e mães do Grupo 2. 


\section{PRÁTICAS EDUCATIVAS PARENTAIS: AUTONOMIA E EXPRESSÃO DE AFETO}

A ANOVA também indiciou diferença estatisticamente significativa entre os grupos na dimensão Autonomia $(F(119)=5,37 ; p<0,01)$. Tal dimensão refere-se ao estimulo e encorajamento que os pais oferecem a seus filhos em relação a autonomia (como tomar decisões, por exemplo). De forma que, a partir da análise de tal dimensão, pode-se compreender que os pais do Grupo 1 fornecem maior estimulo a autonomia que os pais do Grupo 2.

0 incentivo a autonomia contribui para o desenvolvimento de um adulto confiante, seguro e autossuficiente (Weber, 2009), de forma que é possível compreender que a sua ausência não proporcionará base adequada para tal desenvolvimento. Associado a tal fato, Montobbio e Lepri (2007) pontuam a infantilização a qual é sujeita a pessoa com deficiência intelectual e a dificuldade que os pais apresentam em auxiliar o filho a se distanciar e a adquirir seu próprio espaço. Para Franco (2016) é preciso uma reidealização na estética, competência e futuro.

Arthen (2017) defende a necessidade de incentivo à autonomia da pessoa com deficiência, dado que ela é uma construção cotidiana que se dá através de tomada de decisão, vivência de novas experiências, e realização de escolhas cada vez mais complexas. A autora explica que a autonomia é um passo para que a pessoa com deficiência possa advogar a favor de si própria, defendendo seus direitos e exercendo sua cidadania. Rosa e Denari (2013) também relacionam o desenvolvimento de autonomia com a possibilidade de vivências adultas, mais especificamente no que se refere ao exercício de trabalho, para pessoas com deficiência. Os autores pontuam o cuidado que se deve ter enquanto família para que não sejam usadas ao longo do desenvolvimento da pessoa estratégias de superproteção, que tem como foco somente o cuidado com a pessoa com deficiência.

Ainda for observada diferença estatisticamente significativa na dimensão Expressão de Afeto $(F(119)=6,10 ; p<0,01)$, sugerindo que os participantes do Grupo 2 tem maior dificuldade em proporcionar espaço para fala de sentimentos, assim como em relação a expressão do mesmo. Tal característica merece atenção, uma vez que, de acordo com Weber (2017), a expressão de afeto é uma prática que representa o envolvimento dos pais com a vida dos filhos, e está relacionada com melhor autoestima dos filhos. Franco (2016) pontua também que para o desenvolvimento da criança é preciso que haja inclusão primeira na família, ou seja, que haja um processo de ligação e de envolvimento entre os pais e a criança com atraso significativo no desenvolvimento. 0 autor refere também a importância de os pais poderem re-idelizar a criança com deficiência dada a importância de tal feito tanto para o desenvolvimento da própria criança como para que os pais possam exercer de fato seus papéis parentais, e não manterem-se exclusivamente como cuidadores.

Diante dos dados obtidos através desta pesquisa, observa-se que os pais do Grupo 2 fazem uso de estratégias que não incentivam a autonomia, com menor controle autoritário e supervisão dos filhos. Tais características são observadas na literatura (Piccini et al, 2003; Dessen e Silva, 2006) e podem se referir a pais com perfil predominantemente autoritário, práticas que, segundo Decoviv (1989), podem resultar em crianças negligenciadas, rejeitadas ou superprotegidas.

Silva e Dessen (2014), ao estudarem grupo de crianças com deficiências de diferentes qualidades, também observaram o predomínio de uso de práticas coercitivas. As autoras sugeriram relação de tal prática parental devido à severidade da deficiência e as dificuldades advindas delas; assim como ao maior contato com vivências estressantes. Característica também presente em outros estudos sobre 0 alto nível de estresse em pais de crianças com deficiência (Minetto et al, 2012; Kiani, Khodabakhsh e Hasjin, 2016; Craig et al, 2016) assim como acumulo de tarefas parentais (Kiyani et al 2017) que repercutem no tempo e disposição dos pais para interação com seus filhos.

De acordo com Franco (2016) quando os pais não estão disponíveis emocionalmente para seus filhos 0 desenvolvimento da criança é prejudicado. 0 autor defende que a criança é permeada por todas as interações que ocorrem a sua volta, em especial em sua família. De forma que os pais e 
mães que permanecem na negação, na raiva, na culpa, no sofrimento, não conseguem auxiliar adequadamente na promoção do desenvolvimento da criança. Em consonância, Silva e Dessen (2014) pontuam que a deficiência em si deve ser compreendida como um fator de risco para o desequilíbrio familiar. 0 que definirá a ausência ou não de tal desiquilíbrio é a forma como a família estabelecerá suas relações, tanto no momento do diagnóstico como ao longo da vida.

Em revisão de literatura realizada por Rooke, Almeida e Mejía (2017) sobre intervenção com famílias de pessoas com deficiência intelectual identificou o tema "promoção do funcionamento familiar saudável" como mais frequente das intervenções das fontes consultadas (62,5\%); confirmando o papel de destaque que o funcionamento da família precisa ter tanto no que se refere a intervenções como ao desenvolvimento da pessoa com deficiência.

\section{CONCLUSÃO}

Os resultados obtidos na pesquisa permitiram concluir que há diferenças estatisticamente significativas entre práticas educativas parentais utilizadas por pais de crianças com desenvolvimento típico e pais de crianças com atraso de desenvolvimento, no que se refere a expressão de afeto e incentivo a autonomia. Sendo que o segundo grupo faz menor uso de controle autoritário, realiza pouco incentivo a autonomia, e proporciona menos expressões de afeto.

Os achados da pesquisa proporcionam duas reflexões importantes para o desenvolvimento da pessoa com atraso de desenvolvimento. A primeira refere-se ao desenvolvimento da autonomia, fator que pode ser compreendido como uma ponte para a vivência da adultez; que conforme descrito no texto, nem sempre é possível para as pessoas com deficiência. A segunda reflexão diz respeito as relações parentais estabelecidas nas famílias com filhos com atraso significativo no desenvolvimento; e a importância do afeto na promoção do desenvolvimento. Sugerindo um campo de cuidado para além da deficiência em si; paradigma predominante na área de intervenção de pessoas com deficiência.

Além da realização de outros estudos com amostras maiores, é importante que novas investigações sejam realizadas, a fim de identificar quais os fatores da relação parental de pais com filhos com deficiência contribuem para o pouco uso das estratégias supracitadas. Assim como sejam realizados mais estudos que envolvam a temática de práticas educativas parentais e famílias com filhos com deficiência.

Considera-se ainda importante que sejam realizadas ações de orientação e suporte ao pais, proporcionando espaços de construção de práticas educativas mais adequadas, e também que incentivem aumentar a qualidade na relação parental.

\section{REFERÊNCIAS}

Arten, M. N. R. (2017). Autodefesa da pessoa com deficiência: um processo de autonomia e participação. Disponível em: https://www.google.com/url?hl=pt-BR\&q=http://www.portalacesse .com/2017/07/04/autodefesa-da-pessoa-com-deficiencia-um-processo-de-autonomia-e-participacao/\&source=gmail\&ust=15 23439542349000\&usg=AFQjCNF9ftztAURaZaVshRPvD6qH$\mathrm{zfpHg}$

Baumrind, D. (1966). Effects of authoritative control on child behavior. Child Development, 37, 887907.

Bronfenbrenner, U. (2011). Bioecologia do desenvolvimento humano tornando os seres humanos mais humanos. Porto Alegre: Artmed.

Buscaglia, L. (2006). Os deficientes e seus pais. Rio de Janeiro: Editora Record.

Craig, F., Operto, F. F., Giacomo, A., Margari, L., Frolli, A., Conson, M., Ivagnes, S., Monaco, M., 


\section{PRÁTICAS EDUCATIVAS PARENTAIS: AUTONOMIA E EXPRESSÃO DE AFETO}

Margari, F. (2016). Parenting stress among parentes of children with Neurodevelopmental Disorders. Psychiatry Research,242, 121-129.

Darling, N.; Steinberg, L. (1993). Parenting style as contexto: na integrative model. Psychological Bulletin, v. 113, 487-496.

Dekovic, M. (1989). Measuring dimensions and patterns of child rearing: Dutch Version of Block Child Rearing Practices Report (CRPR). Interne Publicatie E. P., Katholieke Universiteit Nijmegen, Netherlands.

Dekovic, M., Janssens, J. M. A.; Gerris, J. R. M. (1991). Factor structure and construct validity of the block child rearing practices report (CRPR). Psychological Assessment: A Journal of Consulting and Clinical Psychology, 3(2), 182-187.

Fiamengui, G. A.; Messa, A. A. (2007). Pais, filhos e deficiência: estudos sobre as relações familiares. Psicologia Ciência e Profissão, (27) 2, 236-245.

Franco, V. (2015). Introdução à intervenção precoce no desenvolvimento da criança: em família, na comunidade, em equipe. Portugal: Edições Aloendro.

Franco, V. (2016). Tornar-se pai/mãe de uma criança com transtornos graves do desenvolvimento. Educar em Revista, Curitiba, Brasil, 59, 35-48.

Gil, A. C. Tipos de Pesquisa. (2008) UFSC. Recuperado em 12 de outubro, 2017, de: https://g00.gl/0fWqyb

Kiyani, A., Fatehi, F., Rasafiani, M., Moradzadeh, R. (2017). Parenting role's tasks as parentes of healthy and disabled children. Internacional Journal of Pediatrics, (5) 9, 5779-5787.

Maccoby, E. \& Martin, J. (1983). Socialization in the context of the family: Parent-child interaction. In E.M. Hetherington (Org.), Handbook of child psychology v. 4 (pp. 1-110). New York: Wiley.

Minetto, M. F. (2010). Práticas educativas parentais, crenças parentais, estresse parental e funcionamento familiar de pais de crianças com desenvolvimento típico e atípico. Tese (Doutorado) Pós-Graduação em Psicologia na Universidade Federal de Santa Catarina. Florianópolis.

Minuchin, S. (1982). Famílias: funcionamento e tratamento. Porto Alegre: Artes Médicas.

Minuchin, S.; Fischman, S. C. (1990). Técnicas de terapia familiar. Porto Alegre: Artes Médicas.

Montobbio, E.; Lepri, C. (2007). Quem eu seria se pudesse ser: a condição adulta da pessoa com deficiência intelectual. Campinas, SP: Fundação síndrome de Down.

Organização Pan-Americana Da Saúde. (2005). Manual para vigilância do desenvolvimento infantil no contexto da AIDPI. Washington. Disponível em: < http://www.bvsde.paho.org /bvsacd/cd61/vigilancia.pdf >

Rooke, M. I.; Almeida, B. R., Mejía, C. F. (2017). Intervenção com famílias de pessoas com deficiência intelectual: análise de produção científica. Revista de Psicologia, Fortaleza, (8) 2, 92-100.

Rosa, F. D.; Denari, F. E. (2013). Trabalho, educação e família: perspectivas para a pessoa com deficiência intelectual. Revista Educação Especial, (26), 45, p. 73-90.

Sampieri, H. E., Collado, F. C., \& Lucio. B. P. M. (2013). Metodologia de pesquisa. Porto Alegre: Penso.

Silva, S. C.; Desse, M. A. (2014). Relações familiares na perspectiva de pais, irmãos e crianças com deficiência. Ver. Bras. Ed. Esp., Marília, (20) 3, 421-434.

Stasiak, G. R.; Weber, L. N. D.; Tucunduva, C. (2014). Qualidade na Interação Familiar e Estresse Parental e suas Relações com o Autoconceito, Habilidades Sociais e Problemas do Comportamento. PSICO, Porto Alegre, (45) 4, 494-501.

Szymanski, H. (2004). Práticas educativas familiares: a família como foco de atenção psicoeducacional. Rev. Estudos de Psicologia, PUC-Campinas, (21) 2, 5-16.

Weber, L. N. D.; Selig, G. A,; Bernardi, M. G.; Salvador, A. P. V. (2006). Continuidade dos estilos 
parentais através das gerações - transmissão intergeracional de estilos parentais. Paidéia, (35) 16, 407-414.

Weber, L. N. D. (2009). Eduque com carinho. Curitiba: Juruá.

Weber, L.N.D. (2017). Relações entre práticas educativas parentais percebidas e a autoestima, sinais de depressão e o uso de substâncias por adolescentes. International Journal of Developmental and Educational Psychology, 1, p. 157-168. 
